\title{
Etiology and treatment alternatives in tooth agenesis: a comprehensive review
}

\author{
Nehir Bilgin, Burçak Kaya \\ Department of Orthodontics, Faculty of Dentistry, Baskent University, Ankara 06490, Turkey.
}

Correspondence to: Dr. Burcak Kaya, Department of Orthodontics, Faculty of Dentistry, Baskent University, Dis Hekimligi Fakultesi, Ortodonti Anabilim Dali, 1. Cad No: 107, Ankara 06490, Turkey. E-mail: burcak_kaya@hotmail.com

How to cite this article: Bilgin N, Kaya B. Etiology and treatment alternatives in tooth agenesis: a comprehensive review. Stomatological Dis Sci2018;2:9. http://dx.doi.org/10.20517/2573-0002.2018.11

Received: 16 Apr 2018 First Decision: 10 Jun 2018 Revised: 21 Aug 2018 Accepted: 7 Nov 2018 Published: 26 Nov 2018

Science Editor: Nikolaos G. Nikitakis Copy Editor: Cui Yu Prduction Editor: Huan-Liang Wu

\begin{abstract}
The purpose of this review article is to examine the etiology, clinical properties and treatment alternatives of tooth agenesis. A comprehensive literature review is realized by using widespread database sources. Disorders in early stages of dental formation may cause congenital or developmental absence of teeth. Both genetic and environmental factors are responsible for tooth agenesis. Skeletal and dental anomalies accompany absence of teeth most of the time. Management of tooth agenesis can be realized by either closing or opening the spaces of congenitally missing teeth and by correction of dentoskeletal problems with orthodontic mechanics. Restorative dentistry procedures accompany orthodontic treatment when filling the spaces of missing teeth or when reshaping the teeth substituting missing teeth. As a conclusion, treatment of problems related with mild or severe tooth agenesis requires multidisciplinary treatment approaches. Early diagnoses of congenitally missing teeth and comprehensive treatment planning have great importance in multidisciplinary approach to prevent or reduce complications which cause both aesthetic and functional disorders and decrease quality of life.
\end{abstract}

Keywords: Tooth agenesis, hypodontia, oligodontia, genetic, space closure, space opening

\section{INTRODUCTION}

Disorders in early stages of tooth formation may cause developmental problems or congenital absence of one or more teeth ${ }^{[1]}$. Congenital absence of at least one tooth is a common dental anomaly ${ }^{[2,3]}$. This definition refers to the word "hypodontia" in the medical terminology which originates from Greek, with "hypo" meaning less and "odous" meaning tooth. The etiology of congenital tooth agenesis is not fully understood, but it is thought to be multifactorial where the roles of many genetic and environmental factors contribute ${ }^{[4]}$. Apart from these, various syndromes, cleft lip and palate, congenital deformities and some systemic diseases were reported in the literature that could lead to tooth agenesis ${ }^{[5]}$. Congenital

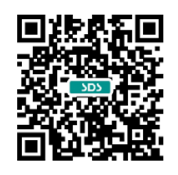


tooth agenesis requires a multidisciplinary treatment approach in which orthodontists, pediatric dentists, prosthodontists, oral and maxillofacial surgeons, laboratory technicians, clinical geneticists, dermatologists, speech and language therapists work as a team ${ }^{[6]}$. The purpose of this review article is to examine the etiology, clinical properties and treatment alternatives of tooth agenesis.

The search strategy was organized across 4 electronic databases (Pubmed, Web of Science, Medline, Scopus) using search terms such as "hypodontia", "oligodontia", "tooth agenesis" and treatment methods such as "space closure" and "space opening". Abstracts for all relevant terms were retrieved and evaluated independently by 2 reviewers (Bilgin N and Kaya B). Exclusion criteria were developed for study selection. Studies published before 1985, involving treatment of tooth loss with etiology other than hypodontia and not available in full text in English or the authors' native language were excluded. Data selection was completed by one reviewer (Bilgin N) and checked for accuracy and consistency by a second reviewer (Kaya B). No reference management software was used and deduplication of literature was checked manually.

\section{CLASSIFICATION OF CONGENITAL TOOTH AGENESIS}

There are many different classifications for congenital tooth absence in the literature. Some researchers classify according to hereditary form, some according to the number of missing teeth and some classify depending on the severity. Usually the third permanent molars are not taken into account when assessing the presence and severity of tooth agenesis. Accordingly, the developmental absence of one or more teeth excluding the third molar teeth is defined as "hypodontia" ${ }^{[1]}$. Some other researchers suggested that the absence of one to six teeth should be called "hypodontia", and the absence of more than six teeth should be called "oligodontia" ${ }^{\text {, } 7,8]}$. In order to reflect the genetic or morphological differences in terminology it was suggested to use subsections like isolated hypodontia or isolated oligodontia for non-syndromic cases and syndromic hypodontia or syndromic oligodontia for cases related to syndromes ${ }^{[7,9]}$. Some researchers graded the severity of congenital tooth absence to help diagnostic classification. Accordingly, the absence of $1-2$ teeth is mild, that of 3-5 teeth is moderate and 6 or more teeth is noted as severe hypodontia ${ }^{[10]}$.

\section{ETIOLOGY OF CONGENITAL TOOTH AGENESIS}

The etiology of congenital tooth agenesis is classified as general and local factors. General factors are a number of genetic conditions such as Down syndrome, cleft lip and palate, ectodermal dysplasia. Local factors are conditions such as trauma to the tooth germ in the early stages of development, hormonal conditions, radiation, infectious diseases and the unintentional removal of tooth germ ${ }^{[6]}$. Diseases such as syphilis, birth injuries, and illnesses the mother has during pregnancy are also contributing factors ${ }^{[10]}$.

In addition to the familial nature of hypodontia, it is thought that this condition may occur as a result of a genetic mutation without a family histor ${ }^{[11]}$. It is not surprising to see problems resulting in dental agenesis in the complex process of tooth formation which is defined as odontogenesis. During odontogenesis, the epithelial-mesenchymal signal at the molecular level is under the control of the wingless (Wnt), hedgehog, fibroblast growth factor and bone morphogenic protein gene family members. Defects seen in any of these pathways may cause problems with tooth morphology (tooth size or shape), tooth mineralization and number of teeth (hypodontia or supernumerary teeth) ${ }^{[10]}$. Studies have shown that the interactions of mutations in the MSX1 and PAX9 homeobox genes during odontogenesis are associated with dental agenesis in mice and may be associated with hypodontia in humans. The PAX9 gene is expressed in mesenchymal elements of developing tooth germ and is required in subsequent stages of dental development. It was observed that tooth growth stagnated in the bud stage in mice with mutant $P A X 9$ gene. It is proven that mutations in MSX1, PAX9, EDA1, WNT10A and EDARDD genes are responsible for isolated hypodontia cases. It is also shown that hypodontia is associated with the AXIN2 gene which is 
responsible for increased colorectal cancer risk ${ }^{[12]}$. The specific mutations of four genes (EDA 1, EDARADD, $E D A R$ and $W N T 10 A$ ) which are effective in $90 \%$ of ectodermal dysplasia cases are demonstrated in the etiology of oligodontia and hypodontia ${ }^{[12]}$.

Infections of tooth germ, trauma or traumatic removal of the deciduous teeth are the major environmental factors for tooth agenesis. It is also reported that exposure to some systemic diseases like syphilis, scarlet fever, rickets during pregnancy and infancy are influential in tooth agenesis. Smoking in pregnancy period or exposure to radiation at a young age may cause problems in formation of some glands and teeth. Chemotherapy and especially radiotherapy affect teeth in an irreversible way ${ }^{[6]}$.

Some syndromes are seen as one of the properties of hypodontia and many gene defects were shown in these syndromes ${ }^{[10]}$. Syndromes like Rieger syndrome, Down syndrome, facial clefts, cleft lip and palate and ectodermal dysplasia are associated with hypodontia ${ }^{[10]}$. In a study conducted on 146 children with nonsyndromic Pierre Robin sequence, it was reported that bilateral absence of second premolars in mandible was the dominant pattern of tooth agenesis ${ }^{[13]}$.

\section{PREVALENCE OF CONGENITAL TOOTH AGENESIS}

Tooth agenesis is rarely seen in deciduous dentition although there is a relationship between hypodontia in deciduous and permanent teeth. Children showing absence in deciduous teeth are reported to show absence in permanent teeth substituting these teeth. In a study, hypodontia in deciduous dentition showed less than $1 \%$ prevalence in Caucasians, while a much higher prevalence was reported in the Japanese population. Deciduous maxillary lateral and mandibular central incisors represent $90 \%$ of the affected deciduous teeth ${ }^{[14]}$.

An examination of the literature reveals that prevalence of hypodontia in permanent dentition increases over time and varies between $1.6 \%$ and $36.5 \%$ depending on the investigated population ${ }^{[14]}$. In another study, congenitally missing teeth prevalence varies between $3.9 \%$ and $6.9 \%$ and is observed slightly more in women than in $\operatorname{men}^{[8]}$. A meta-analysis investigating the prevalence of non-syndromic tooth agenesis which included 33 studies from different regions of the world showed that hypodontia prevalence in Europe was 5.5\% higher and Australia was 6.3\% higher compared to the prevalence in North America which was $1 \%{ }^{[8]}$. Endo et al. ${ }^{[15]}$ reported that $8.5 \%$ of Japanese patients had hypodontia and there was no significant difference between sexes. The average number of missing teeth per individuals presenting hypodontia was 2.4 in Japanese patients. Hypodontia was observed with one or two missing teeth in $76.3 \%$ of individuals, while the prevalence of severe hypodontia -namely oligodontia- was seen in $10.1 \%$ of individuals in Japanese population ${ }^{[15]}$. Two different studies conducted on Turkish population reported that hypodontia prevalence was $4.6 \%$ and $2.8 \%$ respectively ${ }^{[16,17]}$. On the other hand hypodontia prevalence in Irish population was reported to be $11.3 \%$. Hence, the prevalence of tooth agenesis varies greatly between populations ${ }^{[17]}$.

The absence of anterior teeth in mild hypodontia is the dominant pattern, while severe hypodontia is characterized by the absence of posterior teeth ${ }^{[15]}$. Polder et al. ${ }^{[8]}$ revealed that bilateral agenesis is more frequently seen in maxillary lateral incisors. The most frequent unilateral agenesis is observed in mandibular second premolars ${ }^{[8]}$. The most frequent tooth absence patterns within Turkish population are maxillary lateral incisors, mandibular second premolars and mandibular central incisors ${ }^{[16]}$. The Chinese population has a different tooth absence pattern and the most affected teeth are mandibular central incisors followed by maxillary second premolars and mandibular lateral incisors. In Japanese population, the most common missing teeth are mandibular second premolars $(23.7 \%)$ followed by maxillary second premolars (21.5\%), maxillary lateral incisors (17.2\%) and mandibular central incisors (14.0\%). In some researches, 
congenital absence of maxillary permanent canine was detected but it was reported to be rare ${ }^{[6]}$.

\section{SKELETAL AND DENTAL ANOMALIES SEEN IN HYPODONTIA}

Severe hypodontia has been reported to cause a decrease in quality of life associated with oral health. Unfavorable aesthetics is the most common complaint of hypodontia patients. Aesthetic problems caused by hypodontia are dependent on number of missing teeth, size and shape of remaining teeth, size of jaws and position of edentulous spaces. Despite it is a less common complaint stated by patients with hypodontia, missing teeth may also cause difficulties with chewing and speaking ${ }^{[10]}$.

There are several reasons for a possible relationship between congenital absence of teeth and facial skeletal pattern. Neural crest cells play a critical role in the formation of dentoskeletal structures in facial area. Therefore, the skeletal pattern may develop differently in patients with congenitally missing teeth. In addition, according to Moss's functional matrix concept, bone grows by responding to functional relationships created by functional units. Teeth serve as a functional unit in the process of jaw growth. Therefore, the absence of tooth germs may cause developmental deficiencies of apical bone. Dentofacial structure exhibits a functional compensation by showing a different growth pattern in individuals with severe hypodontia ${ }^{[18]}$.

The cephalometric values of patients with congenitally missing teeth show a significant difference in anteroposterior dimensions compared to the norms ${ }^{[18]}$. It is observed that jaws are in a slightly posterior position in patients with hypodontia. Therefore, patients with missing teeth have decreased vertical facial dimensions with a flat and retrognathic profile in general. Upper and lower incisor teeth are significantly more retroclined compared to control groups in patients with congenitally missing teeth. This situation is more severe in people with 10 or more missing permanent teeth. This dentofacial structure caused by hypodontia is perceived much more unaesthetic, since a fuller profile is perceived as aesthetic recently ${ }^{[18]}$.

Tavajohi-Kermani et al. ${ }^{[3]}$ determined a significant correlation between maxillary congenital tooth agenesis and decreased maxillary jaw size. A significant reduction in mandibular corpus size was also shown in a small number of patients with congenitally missing teeth ${ }^{[3]}$.

In the study of Ogaard and Krogstad ${ }^{[19]}$, it was reported that Sella-Nasion-A point angle (SNA) decreased significantly due to maxillary retrusion in moderate and severe hypodontia cases. This situation also caused a decrease in A point-Nasion-B point angle (ANB) and flattened the facial profile. Although there was a significant decrease in lower anterior facial height in the hypodontia group, there was no significant difference in upper anterior facial height. Severe hypodontia typically caused an anterior rotation of mandible as a result of maxillary deficiency and less posterior dental support. Within hypodontia patients, the anterior facial height was shorter in patients with more missing teeth ${ }^{[19]}$.

Vucic et al. ${ }^{[20]}$ observed that anterior hypodontia was significantly correlated with hyperdivergent craniofacial model. A tendency towards class III malocclusion was detected in patients with maxillary hypodontia. A significant decrease in lower anterior facial height was observed in children with posterior mandibular hypodontia. A common characteristic for all types of hypodontia was also shown, which is a significant increase in interincisal angle due to retroclination of maxillary and mandibular incisor teeth $^{[20]}$. Another study showed that intercanine and intermolar widths in maxillary and mandibular arches were significantly smaller in patients with hypodontia compared to the control group. Furthermore, the prevalence of missing teeth was found to be significantly lower in patients with skeletal class 2 pattern. No significant difference was observed in the vertical dimensions of patients with hypodontia ${ }^{[16]}$. 
Bu et al. ${ }^{[21]}$ reported that both maxillary and mandibular arch lengths were significantly shorter in patients with oligodontia. Moreover, intercanine and intermolar widths were significantly reduced in comparison with the control group ${ }^{[21]}$.

\section{TOOTH SIZE AND SHAPE ANOMALIES IN HYPODONTIA}

Microdontia is a condition characterized by smaller than normal teeth and is a widely reported feature of hypodontia. In addition to their small sizes, the affected teeth usually have abnormal contoured crowns. The affected lateral incisors have either an oval shape or a conical crown which gets thinner from cervix to incisal edge. The roots of these teeth are almost shrunk and root formation abnormalities may be observed additionally. Ectopic eruption of permanent teeth is common in hypodontia cases ${ }^{[10]}$. A study showed a significant relationship between missing maxillary lateral incisors and positions of other permanent teeth. A similar relationship is also found between maxillary lateral microdontia and palatinal positioning of canines or distal angulation of mandibular secondary premolars ${ }^{[22]}$.

Root resorption of a deciduous tooth is usually delayed if there is no permanent tooth bud under it. Persistent deciduous teeth can provide a satisfactory service for years, although significant amounts of root resorptions are observed. However, they often remain in infraocclusion due to localized insufficiency of alveolar development and relatively excessive eruption of adjacent teeth. As a result, persistent deciduous teeth generally become ankylosed ${ }^{[10]}$.

Another feature of hypodontia is the delay of tooth eruption. Tunç et al ${ }^{[23]}$ reported that children with mild to moderate hypodontia showed a significant delay of 0.3 years in dental development in both sexes. No correlation was observed between dental and chronological age or the severity of hypodontia ${ }^{[23]}$.

\section{TREATMENT OPTIONS IN CONGENITAL TOOTH AGENESIS}

Comprehensive treatment covering a long period of time must be planned in hypodontia cases to achieve optimum results for a lifetime. A treatment plan may be prepared depending on the patient's age, complaints and dental development ${ }^{[10]}$.

\section{SPACE CLOSURE WITH ERUPTION GUIDANCE}

Guidance of eruption described by Hotz can be applied in congenital absence of maxillary incisor teeth during early mixed dentition stage in children who have tendency to increased overjet, openbite, protrusive incisors, crowding. This method makes spontaneous closure of gaps belonging to congenitally missing teeth possible by erupting maxillary canines in place of maxillary lateral incisors and achieving a class II occlusion. Enamel reduction must be applied in maxillary 1st and 2nd deciduous molars on mesial and distal sides for this purpose. In case of congenital maxillary or mandibular 2nd premolars absence, then 1st deciduous molars should be reduced from distal side and 2 nd deciduous molars should be reduced from mesial and distal sides, if it is aimed to close the spaces of missing teeth with eruption guidance. Alternatively, eruption guidance can be conducted with early removal of maxillary and mandibular 1st and 2nd deciduous molars ${ }^{[24]}$.

The purpose of arrangements in edentulous spaces with orthodontic mechanics is to create an ideal sized area for denture prosthesis, implants or the build-up restorations of microdontic teeth. Fixed orthodontic appliances which allow parallel movement are more preferable for this purpose and adequate root parallelism is ensured ${ }^{[10]}$.

Congenital lateral absence is one of the most common dental agenesis. One of the treatment alternatives is opening of spaces and then applying prosthetic restorations. The other option is closing of spaces with 

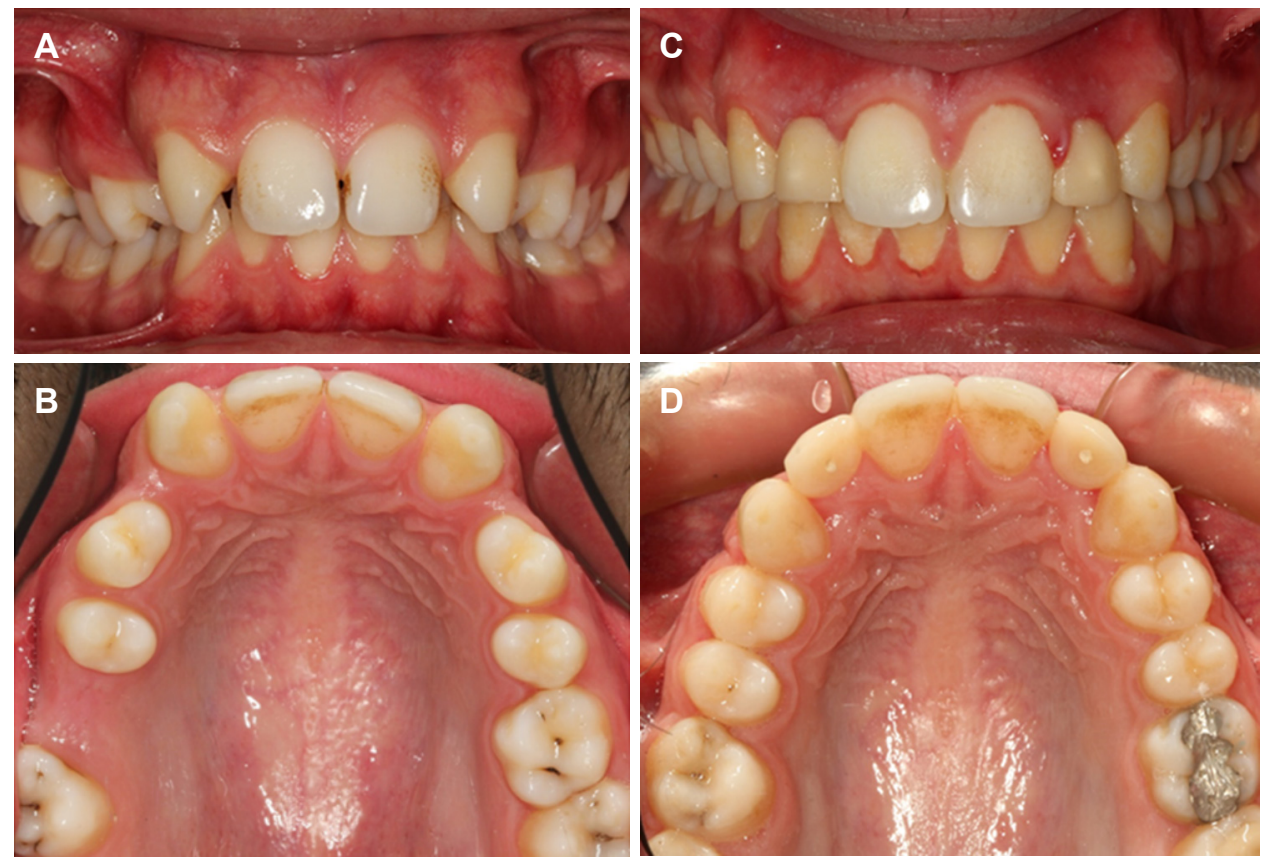

Figure 1. Management of bilateral maxillary lateral incisor absence with space opening. A: Intraoral frontal view before treatment; B: intraoral occlusal view before treatment; $\mathrm{C}$ : intraoral frontal view after completion of orthodontic treatment. Temporary crowns are placed on dental implants bilaterally for substitution of lateral incisors; D: intraoral occlusal view after completion of orthodontic treatment. Spaces are opened for dental implants for substitution of missing lateral incisors, whereas the space of missing right first molar is closed

orthodontics followed by recontouring of canines and premolars. It is suggested that patients' age, type of skeletal and dental malocclusion in sagittal and vertical dimensions, crowding in both dental arches and facial profile should be analyzed before choosing the best treatment alternative ${ }^{[25]}$. Overjet, overbite and posterior occlusion must be evaluated carefully while deciding to open or close the spaces of congenitally missing teeth in alveolar bone. Spaces had better opened if there is a favorable molar relationship, a decent overjet or a deepbite. Additionally, when a tooth is planned to substitute another tooth, then parameters like tooth size, shape, color and eruption level have to be evaluated ${ }^{[10]}$.

\section{ORTHODONTIC SPACE OPENING FOLLOWED BY PROSTHETIC TREATMENT}

If the treatment plan involves opening spaces for missing teeth, then these spaces may be replaced with resin bonded fixed partial prosthetic restorations, conventional fixed partial prosthetic restorations, implant supported prosthetic restorations or modified adhesive partial prosthetic restorations ${ }^{[10]}$.

There are three ways to determine the proper space for missing lateral incisors. These are using the golden ratio, Bolton analysis or the mesiodistal size of contralateral lateral incisor tooth [Figure 1] . Golden ratio or Bolton analysis can be used for measurement of edentulous spaces in patients with either unilateral or bilateral congenitally missing maxillary lateral incisors ${ }^{[26]}$.

\section{Resin bonded fixed partial dentures}

Resin bonded fixed partial dentures can be used for replacement of only a few missing teeth. The most conservative tooth supported prosthetic treatment approach is a resin bonded fixed partial denture, because adjacent teeth remain almost untouched. The thickness and translucency of supporting teeth have an important effect on the design of resin bonded fixed partial dentures. If the metal retainer unit is positioned too much incisally, it may cause a grey reflection through a highly translucent adjacent teeth in the incisal third ${ }^{[26]}$. 


\section{Conventional fixed partial dentures}

A less conservative tooth supported restoration type is a conventional fixed partial denture. The major disadvantage of these dentures is the required preparation of the adjacent teeth. Fixed partial dentures are not the best treatment options for young patients who have congenitally missing maxillary lateral incisors due to preparation requirement ${ }^{[2]}$. Patients with teeth supported fixed partial dentures are reported to have unfavorable periodontal conditions compared to patients who have only natural teeth after orthodontic treatment with space closure ${ }^{[25]}$.

Important issues while planning these restorations are the aligning and leveling of adjacent teeth. The orthodontist should carefully evaluate the inclination and angulation of central incisor and canine teeth during the process of aligning ${ }^{[26]}$.

\section{Implant supported dentures}

The spaces in anterior and posterior regions may be replaced with implant supported dentures after teeth adjacent to edentulous spaces are leveled with orthodontic treatment ${ }^{[27]}$. Dental implants may remain infraocclusally after completion of growth, if they are placed in growing patients. For this reason, dental implant treatments should be delayed after completion of growth ${ }^{[10]}$.

A frequent feature seen in hypodontic patients is that alveolar crest thickness is not adequate in edentulous areas. If the crest width is inappropriate for a dental implant, then the use of small diameter implants or surgical procedures for widening the alveolar crest can be applied. Alternatively, alveolar distraction osteogenesis techniques can be applied if there is enough cooperation by the patient or bone augmentation procedures can be used if needed. Autogenic bone grafts are the gold standard in such situations ${ }^{[10]}$. On the other hand, replacement of missing teeth with dental implants has some disadvantages like accumulation of dental plaque, gingival inflammation and increased treatment $\operatorname{costs}^{[28]}$.

\section{ORTHODONTIC SPACE OPENING FOLLOWED BY AUTOTRANSPLANTATION}

Transplantation of maxillary third molars to edentulous areas or premolar extraction in other quarters of jaws that shows crowding can be applied in congenital absence of second premolars ${ }^{[10]}$. Extraction of the tooth to be transplanted must be achieved without any detriment to root morphology, with the least possible damage to periodontal ligament and root cement by applying a rigorous surgical protocol during autotransplantation. It is ideal to have an open tooth apex that allows pulpal revascularization and three quarters of root length should be developed. Root canal treatment may not be necessary in a tooth with an open apex, because revascularization may occur spontaneously ${ }^{[10]}$. On the other hand, root canal treatment should be performed in an appropriate time when a tooth with a closed apex is transplanted. Orthodontic force should not be applied for the first 3-6 months after autotransplantation. Additionally, the amount and duration of force should be minimized when it is applied. Orthodontic treatment can be initiated after the radiographic verification of lamina dura ${ }^{[10]}$.

Kokai et al. ${ }^{[29]}$ examined the clinical and radiological outcomes early orthodontic forces application (within 4-8 weeks) with $\mathrm{Ni}$-Ti arch wires after autotransplantation. One hundred teeth which had completed their root development during autotransplantation stage were evaluated with regards to root resorption, ankylosis, mobility, pocket depth and inflammation in the tooth receiving area. Root canal treatments of teeth were performed in the second week after transplantation. It was reported that early application of orthodontic forces could increase success rate of the autotransplanted teeth. Besides, it was stated that the type of donor teeth and pre-surgical occlusal condition can affect success rate of transplantation ${ }^{[29]}$. 

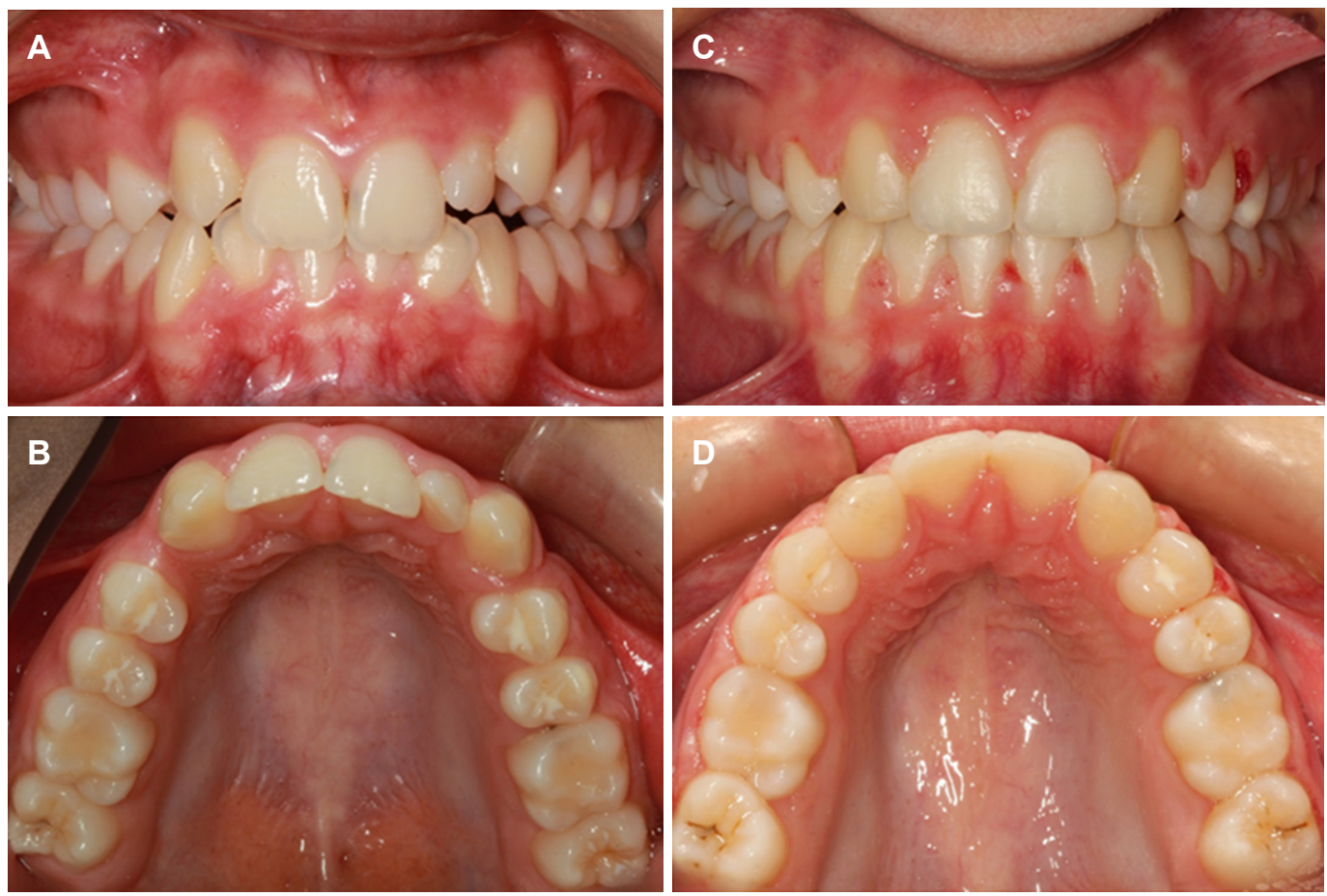

Figure 2. Management of unilateral maxillary lateral incisor absence with space closing. A: Intraoral frontal view before treatment; B: intraoral occlusal view before treatment; C: intraoral frontal view after completion of orthodontic treatment. Canines and first premolars are mesialized bilaterally for substitution of lateral incisors and canines, respectively; D: intraoral occlusal view after completion of orthodontic treatment. The microdontic left lateral incisor is extracted and the spaces of missing lateral incisors are closed bilaterally by mesialization of the posterior segments

\section{ORTHODONTIC SPACE CLOSURE FOLLOWED BY ESTHETIC RESTORATIVE TREATMENTS}

Space closure can be preferred in the absence of maxillary lateral teeth if there is increased overjet, openbite, protrusive incisors, crowding, small/white canines, continuing vertical alveolar growth and if other teeth are healthy [Figure 2]. The molar relationship becomes class II and the occlusion leads to a group function when spaces in maxillary arch are closed with orthodontic treatment in bilateral absence of lateral teeth. Additionally, restorative applications for maxillary canines and premolars for substitution of lateral and canine teeth are required. The main advantage of space closure is that the whole treatment can be finished with orthodontic treatment alone. Besides this treatment approach enables hard and soft tissues to remain in a natural state that can better adapt to changes over time ${ }^{[30]}$.

Deepbite can be observed in hypodontia patients, especially when incisor teeth are missing in mandibular arch. This problem can be eliminated with intrusion of incisors, extrusion of premolars and molars or proclination of incisors in growing patients. Reverse curve arch wires can be used in mandibular arch to obtain extrusion of premolars and molars while incisors can be proclined at the same time. This method does not work in severe cases especially if there are missing lower incisors, premolars, molars and the direction of growth shows anterior rotation in the mandible. In such cases, removable appliances with anterior bite planes can be used in maxillary arch for mandibular molar extrusion in early stages. Alternatively, temporary anchorage devices such as miniscrews can be used to apply intrusive forces on lower incisors ${ }^{[10]}$.

Extrusion of canines with orthodontic treatment enhances gingival height harmony between central incisors and canines in cases where canines will substitute lateral incisors. Ideally, lateral incisors' gingival level should be approximately $1 \mathrm{~mm}$ coronal from central incisors' gingival level. But achieving this relationship is important only for individuals with a high smile line. It should also be noted that enamel 
should be removed from incisal edges of canines after extrusion. This is because incisal edges of lateral teeth should be 0.5-1 mm more apical than incisal edges of central teeth for ideal aesthetics ${ }^{[10]}$. Additionally, uprighting of canines to a fair amount is required for substitution of lateral incisors. Thus, they will appear narrower mesiodistally. Inclination of maxillary canines should also be adjusted so that root appearance is minimized. Bleaching of canines with carbamide peroxide gel of $10 \%-15 \%$ can be recommended in order to obtain an ideal lateral tooth color. Adequate amount of enamel has to be reduced from cusp tips, mesial/ distal edges, buccal contours and singulums for better substitution of lateral teeth ${ }^{[10]}$.

A number of orthodontic modifications may be applied for anterior aesthetics in space closure cases where maxillary first premolars are used for substitution of canines. These modifications are: slight mesiopalatal rotations to conceal premolars' palatinal cusp, intrusion of premolars to adjust gingival margin levels of premolars and central incisors, moving premolars buccally to obtain appearance of canine. It may also be needed to use composite build-up restorations to increase mesiodistal width of premolars. Furthermore, enamel reduction in palatinal cusps of premolars may need grinding. The additional procedures applied to teeth in order to change their colors and shapes, to enlarge their size or to treat structural defects are called adhesive build-up restorations. Composite resins are used for this procedure ${ }^{[10]}$.

Laminate veneer restorations are one of the least invasive therapies that can be used to enhance dental aesthetics. The most successful aesthetic results are achieved with porcelain veneer restorations, but they require more precise techniques than composite veneers. However, composite build-up restorations or composite veneer restorations cannot provide the ideal reflection or translucency similar to enamel. Furthermore, composites are less resistant to breakage. Most studies remarked that porcelain laminate veneer restorations have more than $90 \%$ success. Porcelain laminate veneers are promising permanent treatment options for restoration of anterior teeth, which is required in the treatment of congenital lateral agenesis $^{[10]}$.

\section{CONCLUSION}

The exclusion criteria used in this review were not very strict and allowed inclusion of studies having mixed-method study designs. This situation might have caused contradictions in interpretation of some study outcomes and is the limitation of this review. Congenital tooth agenesis is a common dental anomaly which causes both aesthetic and functional disorders. Early diagnosis of absent teeth is important and comprehensive treatment planning involving correction of skeletal discrepancies, elimination of deepbite, aligning and levelling of teeth and space arrangements is necessary for patients with hypodontia who require multidisciplinary treatment approaches. Future innovations in this field may bring up treatment of the genes causing tooth agenesis with gene therapies and development of tooth tissues from dental stem cells to the agenda.

\section{DECLARATIONS}

\section{Authors' contributions}

Examined the literature, collected the data, wrote and approved the manuscript: Bilgin $\mathrm{N}$

Designed the study, interpreted the data, wrote, revised and approved the manuscript: Kaya B

\section{Availability of data and materials}

Not applicable.

\section{Financial support and sponsorship}

None. 


\section{Conflict of interest}

All authors declared that there are no conflicts of interest.

\section{Ethical approval and consent to participate}

Not applicable.

\section{Consent for publication}

Not applicable.

\section{Copyright}

(C) The Author(s) 2018.

\section{REFERENCES}

1. Taju W, Sherriff M, Bister D, Shah S. Association between severity of hypodontia and cephalometric skeletal patterns: a retrospective study. Eur J Orthod 2017;40:200-5.

2. Rakhshan V, Rakhshan H. Meta-analysis and systematic review of the number of non-syndromic congenitally missing permanent teeth per affected individual and its influencing factors. Eur J Orthod 2016;38:170-7.

3. Tavajohi-Kermani H, Kapur R, Sciote JJ. Tooth agenesis and craniofacial morphology in an orthodontic population. Am J Orthod Dentofacial Orthop 2002;122:39-47.

4. Barros SE, Janson G, Torres FC, de Freitas MR, de Almeida RR. Class I malocclusion treatment: Influence of a missing mandibular incisor on anterior guidance. Am J Orthod Dentofacial Orthop 2010;138:109-17.

5. Graber LW. Congenital absence of teeth: a review with emphasis on inheritance patterns. J Am Dent Assoc 1978;96:266-75.

6. AlShahrani I, Togoo RA, AlQarni MA. A review of hypodontia: classification, prevalence, etiology, associated anomalies, clinical implications and treatment options. World J Dent 2013;4:117-25.

7. Arte S, Pirinen S. Hypodontia. Available from: https://www.orpha.net/data/patho/GB/uk-hypodontia.pdf. [Last accessed on 9 Nov 2018]

8. Polder BJ, Van't Hof MA, Van der Linden FP, Kuijpers-Jagtman AM. A meta-analysis of the prevalence of dental agenesis of permanent teeth. Community Dent Oral Epidemiol 2004;32:217-26.

9. Schalk-van der Weide Y, Steen WH, Bosman F. Distribution of missing teeth and tooth morphology in patients with oligodontia. ASDC J Dent Child 1992;59:133-40.

10. Hobkirk JA, Gill DS, Jones SP, Hemmings KW, Bassi GS, et al. Hypodontia: a team approach to management. 1st ed. West Sussex: WileyBlackwell; 2011. pp. 3-27.

11. Kupietzky A, Houpt M. Hypohidrotic ectodermal dysplasia: characteristics and treatment. Quintessence Int 1995;26:285-91.

12. Dreesen K, Swinnen S, Devriendt K, Carels C. Tooth agenesis patterns and phenotype variation in a cohort of Belgian patients with hypodontia and oligodontia clustered in 79 families with their pedigrees. Eur J Orthod 2014;36:99-106.

13. Antonarakis GS, Suri S. Prevalence and patterns of permanent tooth agenesis in patients with nonsyndromic Pierre Robin sequence. Am J Orthod Dentofacial Orthop 2014;145:452-60.

14. Al-Ani AH, Antoun JS, Thomson WM, Merriman TR, Farella M. Hypodontia: an update on its etiology, classification, and clinical management. Biomed Res Int 2017;2017:9378325.

15. Endo T, Ozoe R, Kubota M, Akiyama M, Shimooka S. A survey of hypodontia in Japanese orthodontic patients. Am J Orthod Dentofacial Orthop 2006;129:29-35.

16. Celikoglu M, Kazanci F, Miloglu O, Oztek O, Kamak H, et al. Frequency and characteristics of tooth agenesis among an orthodontic patient population. Med Oral Patol Oral Cir Bucal 2010;15:e797-801.

17. Shimizu T, Maeda T. Prevalence and genetic basis of tooth agenesis. Jpn Dent Sci Rev 2009;45:52-8.

18. Ben-Bassat Y, Brin I. Skeletodental patterns in patients with multiple congenitally missing teeth. Am J Orthod Dentofacial Orthop 2003;124:521-5.

19. Ogaard B, Krogstad O. Craniofacial structure and soft tissue profile in patients with severe hypodontia. Am J Orthod Dentofacial Orthop 1995; 108:472-7.

20. Vucic S, Dhamo B, Kuijpers MA, Jaddoe VW, Hofman A, et al. Craniofacial characteristics of children with mild hypodontia. Am J Orthod Dentofacial Orthop 2016;150:611-9.

21. Bu X, Khalaf K, Hobson RS. Dental arch dimensions in oligodontia patients. Am J Orthod Dentofacial Orthop 2008;134:768-72.

22. Garib DG, Alencar BM, Lauris JR, Baccetti T. Agenesis of maxillary lateral incisors and associated dental anomalies. Am J Orthod Dentofacial Orthop 2010; doi: 10.1016/j.ajodo.2009.12.024.

23. Tunç EŞ, Bayrak S, Koyutürk AE. Dental development in children with mild-to-moderate hypodontia. Am J Orthod Dentofacial Orthop 2011;139:334-8.

24. Ülgen M. Orthodontic Treatment Principles. 7th ed. Ankara: AUDHFY; 2005. pp. 101-2.

25. Silveira GS, de Almeida NV, Pereira DM, Mattos CT, Mucha JN. Prosthetic replacement vs space closure for maxillary lateral incisor agenesis: a systematic review. Am J Orthod Dentofacial Orthop 2016;150:228-37.

26. Kinzer GA, Kokich VO Jr. Managing congenitally missing lateral incisors. Part II: tooth-supported restorations. J Esthet Restor Dent 
2005; 17:76-84.

27. de Avila ÉD, de Molon RS, de Assis Mollo F Jr, de Barros LA, Capelozza Filho L, et al. Multidisciplinary approach for the aesthetic treatment of maxillary lateral incisors agenesis: thinking about implants? Oral Surg Oral Med Oral Pathol Oral Radiol 2012;114:e22-8.

28. Bizetto MS, Tessarollo FR, Jimenez EE, Guariza-Filho O, Camargo ES, et al. Implant rehabilitation of canines in case of bilaterally missing maxillary lateral incisors. Am J Orthod Dentofacial Orthop 2013;144:110-8.

29. Kokai S, Kanno Z, Koike S, Uesugi S, Takahashi Y, et al. Retrospective study of 100 autotransplanted teeth with complete root formation and subsequent orthodontic treatment. Am J Orthod Dentofacial Orthop 2015;148:982-9.

30. Schneider U, Moser L, Fornasetti M, Piattella M, Siciliani G. Esthetic evaluation of implants vs canine substitution in patients with congenitally missing maxillary lateral incisors: are there any new insights? Am J Orthod Dentofacial Orthop 2016;150:416-24. 